\title{
PAX5 NP_057953.1:p.G183S
}

National Cancer Institute

\section{Source}

National Cancer Institute. PAX5 NP 057953.1:p.G183S. NCI Thesaurus. Code C158144.

A change in the amino acid residue at position 183 in paired box protein Pax-5 where glycine has been replaced by serine. 